\title{
Deficient and Ramified Small Functions for Admissible Solutions of Some Differential Equations II
}

\author{
Katsuya ISHIZAKI and Kenji FUJITA \\ Tokyo National College of Technology \\ (Communicated by T. Nagano)
}

\begin{abstract}
Let $\alpha_{j}(z), j=1,2, a_{i}(z), i=1,2, \cdots, 6$ be meromorphic functions. Suppose the differential equation $(*) w^{\prime 3}+\alpha_{2}(z) w^{\prime 2}+\alpha_{1}(z) w^{\prime}=a_{6}(z) w^{6}+\cdots+a_{1}(z) w+a_{0}(z)$ possesses an admissible solution $w(z)$. If $\eta(z)$ is a solution of $(*)$ and small with respect to $w(z)$ and if $(*)$ is irreducible, then $\eta(z)$ is a deficient or a ramified function for $w(z)$.
\end{abstract}

\section{Introduction.}

We use here standard notations in Nevanlinna theory [2][6][8]. Let $f(z)$ be a meromorphic function. In this paper the term "meromorphic" will mean meromorphic in $|z|<\infty$. As usual, $m(r, f), N(r, f)$, and $T(r, f)$ denote the proximity function, the counting function, and the characteristic function of $f(z)$, respectively. Let $\bar{N}(r, f)$ be the counting function for distinct poles of $f(z)$. Put $N_{1}(r, f)=N(r, f)-\bar{N}(r, f)$. For $\alpha \in \mathbb{C}$, the following quantities are defined

$$
\delta(\alpha, f)=\liminf _{r \rightarrow \infty} \frac{m(r, 1 /(f-\alpha))}{T(r, f)} \quad(\text { deficiency })
$$

and

$$
\theta(\alpha, f)=\liminf _{r \rightarrow \infty} \frac{N_{1}(r, 1 /(f-\alpha))}{T(r, f)} \quad \text { (ramification index) }
$$

A function $\varphi(r), 0 \leq r \leq \infty$, is said to be $S(r, f)$ if there is a set $E \subset \mathbb{R}^{+}$of finite linear measure such that $\varphi(r)=o(T(r, f))$ as $r \rightarrow \infty, r \notin E$. A meromorphic function $a(z)$ is said to be small with respect to $f(z)$ if $T(r, a)=S(r, f)$. We consider here the deficiency and the ramification for a small function $a(z)$ instead of complex number $\alpha \in \mathbb{C}$. We put, for a meromorphic function $a(z), m(r, a ; f)=m(r, 1 /(f-a)), N(r, a ; f)=N(r, 1 /(f-a))$, and $\bar{N}(r, a ; f), N_{1}(r, a ; f), \delta(a, f), \theta(a, f)$, etc., are defined in the same way as for a complex number $\alpha \in \mathbb{C}$, respectively. If $\delta(a, f)>0$ or $\theta(a, f)>0$, then $a(z)$ is said to be a 
deficient or ramified function for $f(z)$, respectively.

Let $\mathscr{M}$ be a finite collection of meromorphic functions. A transcendental meromorphic function $w(z)$ is admissible with respect to $\mathscr{M}$, if $T(r, a)=S(r, w)$ for any $a(z) \in$ $\mathscr{M}$. Suppose a transcendental meromorphic function $w(z)$ is admissible with respect to $\mathscr{M}$. For $c \in \mathbb{C} \cup\{\infty\}, z_{0}$ is admissible $c$-point with respect to $\mathscr{M}$, if $z_{0}$ is $c$-point of $w(z)$ and neither zero nor pole of $a(z)$ which belongs to $\mathscr{M}$. Suppose $N(r, c ; f) \neq S(r, f)$, $c \in \mathbb{C} \cup\{\infty\}$. We denote by $n_{\mathbb{C}_{1}}^{*}(r, c ; f)$, the number of $c$-point $z_{0}$ of $f(z)$ in $|z| \leqq r$ so that $z_{0}$ satisfies some condition $\mathrm{C} 1 . N_{C_{1}}^{*}(r, c ; f)$ is defined in the usual way. We use the word "almost all" $c$-point satisfy the condition $\mathrm{Cl}$, if

$$
N(r, c ; f)-N_{\mathrm{C}_{1}}^{*}(r, c ; f)=S(r, f) .
$$

REMARK 1. Let $\mathscr{M}$ be a finite collection of meromorphic functions. Suppose a transcendental meromorphic function $w(z)$ is admissible with respect to $\mathscr{M}$. Let $\eta(z)$ be rational of members of $\mathscr{M}$ and their derivatives. Then we have $T(r, \eta) \leq$ $K \sum_{a_{v} \in \mathscr{M}} T\left(r, a_{v}\right)+S(r, w)$, for some $K>0$. Thus $\eta(z)$ is a small function with respect to $w(z)$. Assume that $N(r, w) \neq S(r, w)$, then there exists an admissible pole of $w(z)$ with respect to $\mathscr{M}$. If $\eta(z)$ vanishes at almost all poles of $w(z)$, then $\eta(z) \equiv 0$.

Let $\Omega\left(z, w, w^{\prime}, \cdots, w^{(n)}\right)$ be a differential polynomial of $w$ with meromorphic coefficients and let $\mathscr{M}$ be the collection of coefficients of $\Omega$. We call $w(z)$ an admissible solution of the equation

$$
\Omega\left(z, w, w^{\prime}, \cdots, w^{(n)}\right)=0,
$$

if $w(z)$ satisfies the above equation and $w(z)$ is admissible with respect to $\mathscr{M}$.

Let $M$ be the field of meromorphic functions and let $\Omega\left(z, w, w^{\prime}\right)$ be a polynomial of $w$ and $w^{\prime}$ with meromorphic (possibly transcendental) coefficients. We call the differential polynomial $\Omega\left(z, w, w^{\prime}\right)$ irreducible, if $\Omega\left(z, w, w^{\prime}\right)$ is irreducible over the field $M$.

We know the following theorem due to Mokhońko [7]:

THEOREM A. Suppose the differential equation (1.1) possesses an admissible solution $w(z)$. If $\eta(z)$ is a deficient or ramified small function for $w(z)$, then $\eta(z)$ is a small solution of (1.1), i.e.

$$
\Omega\left(z, \eta, \eta^{\prime}, \cdots, \eta^{(n)}\right)=0 .
$$

Our aim in this note is to get a converse of this result for the special case of (1.1), that is, for the equation of the form

$$
P\left(z, w^{\prime}\right)=Q(z, w),
$$

where $P\left(z, w^{\prime}\right)$ and $Q(z, w)$ are polynomials of $w^{\prime}$ and $w$ with meromorphic coefficients, respectively. In [3], we obtained the following theorem for the case $p=\operatorname{deg}_{w^{\prime}}\left[P\left(z, w^{\prime}\right)\right]=$ 2 . 
THEOREM B. Suppose the differential equation

$$
w^{\prime 2}+\alpha_{1}(z) w^{\prime}=a_{4}(z) w^{4}+\cdots+a_{1}(z) w+a_{0}(z)
$$

possesses an admissible solution $w(z)$, where the coefficients are meromorphic and $\left|a_{4}\right|+\left|a_{3}\right|+\left|a_{2}\right| \not \equiv 0$. If $\eta(z)$ is a small solution of $(1.3)$, then $\eta(z)$ is a deficient or ramified function of $w$, unless (1.3) is reducible.

In this note, we treat the case $p=3$ in (1.2), and prove the following theorem.

THEOREM 1. Suppose the differential equation

$$
w^{\prime 3}+\alpha_{2}(z) w^{\prime 2}+\alpha_{1}(z) w^{\prime}=a_{6}(z) w^{6}+\cdots+a_{1}(z) w+a_{0}(z)
$$

possesses an admissible solution $w(z)$, where the coefficients are meromorphic and $\left|a_{6}\right|+\left|a_{5}\right|+\left|a_{4}\right|+\left|a_{3}\right| \not \equiv 0$. If $\eta(z)$ is a small solution of $(1.4)$, then $\eta(z)$ is a deficient or. ramified function of $w$, unless (1.4) is reducible.

\section{Preliminary lemmas.}

LEMMA 1 ([5]). Suppose (1.4) possesses an admissible solution $w(z)$. If $w(z)$ satisfies the Riccati equation or the differential equation

$$
w^{\prime 2}+B(z, w) w^{\prime}+A(z, w)=0
$$

where $B(z, w)$ and $A(z, w)$ are polynomials of $w$ with small (w.r.t. $w(z))$ coefficients and $\operatorname{deg}_{w}[B(z, w)] \leq 2, \operatorname{deg}_{w}[A(z, w)] \leq 4$, then (1.4) is reducible.

REMARK 2. Put $y=[a(z) w+b(z)] /[c(z) w+d(z)], a d-b c \neq \equiv 0$ in (2.1), where $a(z)$, $b(z), c(z)$ and $d(z)$ are small (w.r.t. $w(z))$ functions. Then $y(z)$ satisfies the Riccati equation or the differential equation of the form

$$
y^{\prime 2}+\widetilde{B}(z, y) y^{\prime}+\widetilde{A}(z, y)=0
$$

where $\widetilde{B}(z, y)$ and $\widetilde{A}(z, y)$ are polynomials of $y$ with small (w.r.t. $y(z))$ coefficients and $\operatorname{deg}_{y}[\tilde{B}(z, y)] \leq 2, \operatorname{deg}_{y}[\tilde{A}(z, y)] \leq 4$.

The equation (2.1) was treated by Steinmetz in [9] and by Eremenko in [1]. To state Lemma 2, we define some notations (see [4]).

Let $f(z)$ be a transcendental meromorphic function and let $\alpha_{1}(z), \cdots, \alpha_{4}(z), \beta_{1}(z)$, $\cdots, \beta_{4}(z), \gamma_{1}(z), \cdots, \gamma_{4}(z), \delta_{1}(z), \cdots, \delta_{4}(z), \lambda_{1}(z)$ and $\lambda_{0}(z)$ be small functions with respect to $f(z)$, where $\lambda_{1}(z)^{2}-4 \lambda_{0}(z) \not \equiv 0, \quad \alpha_{4}(z)^{2}-\lambda_{1}(z) \alpha_{3}(z) \alpha_{4}(z)+\lambda_{0}(z) \alpha_{3}(z)^{2} \not \equiv 0, \quad \beta_{4}(z)^{2}-$ $\lambda_{1}(z) \beta_{3}(z) \beta_{4}(z)+\lambda_{0}(z) \beta_{3}(z)^{2} \not \equiv 0, \quad \gamma_{4}(z)^{2}-\lambda_{1}(z) \gamma_{3}(z) \gamma_{4}(z)+\lambda_{0}(z) \gamma_{3}(z)^{2} \not \equiv 0, \quad \delta_{4}(z)^{2}-$ $\lambda_{1}(z) \delta_{3}(z) \delta_{4}(z)+\lambda_{0}(z) \delta_{3}(z)^{2} \not \equiv 0$.

Let $z_{0}$ be a simple pole of $f(z)$. We call $z_{0}$ strongly representable in the second kind sense by $\alpha_{1}(z), \cdots, \alpha_{4}(z), \beta_{1}(z), \cdots, \beta_{4}(z), \gamma_{1}(z), \cdots, \gamma_{4}(z), \delta_{1}(z), \cdots, \delta_{4}(z), \lambda_{1}(z)$ and $\lambda_{0}(z)$, if $f(z)$ is written in the neighbourhood of $z_{0}$ as: 


$$
f(z)=\frac{R}{z-z_{0}}+\alpha+\beta\left(z-z_{0}\right)+\gamma\left(z-z_{0}\right)^{2}+\delta\left(z-z_{0}\right)^{3}+O\left(z-z_{0}\right)^{4}
$$

and

$$
\begin{array}{cc}
R^{2}+\lambda_{1}\left(z_{0}\right) R+\lambda_{0}\left(z_{0}\right)=0, & \\
\alpha=\frac{\alpha_{1}\left(z_{0}\right) R+\alpha_{2}\left(z_{0}\right)}{\alpha_{3}\left(z_{0}\right) R+\alpha_{4}\left(z_{0}\right)}, & \beta=\frac{\beta_{1}\left(z_{0}\right) R+\beta_{2}\left(z_{0}\right)}{\beta_{3}\left(z_{0}\right) R+\beta_{4}\left(z_{0}\right)}, \\
\gamma=\frac{\gamma_{1}\left(z_{0}\right) R+\gamma_{2}\left(z_{0}\right)}{\gamma_{3}\left(z_{0}\right) R+\gamma_{4}\left(z_{0}\right)}, & \delta=\frac{\delta_{1}\left(z_{0}\right) R+\delta_{2}\left(z_{0}\right)}{\delta_{3}\left(z_{0}\right) R+\delta_{4}\left(z_{0}\right)} .
\end{array}
$$

For the sake of brevity, we call such simple pole, SS2-kind pole.

LEMMA 2. Let $w(z)$ be a transcendental meromorphic function and let $\alpha_{1}(z), \cdots$, $\alpha_{4}(z), \beta_{1}(z), \cdots, \beta_{4}(z), \gamma_{1}(z), \cdots, \gamma_{4}(z), \delta_{1}(z), \cdots, \delta_{4}(z), \lambda_{1}(z)$ and $\lambda_{0}(z)$ be small functions with respect to $w(z)$. We denote by $n_{\langle\mathrm{SS} 2\rangle}(r, w)$ the number of the SS2-kind poles in $|z| \leqq r$. $N_{\langle\mathrm{SS} 2\rangle}(r, w)$ is defined in terms of $n_{\langle\mathrm{Ss} 2\rangle}(r, w)$ in the usual way. If

$$
m(r, w)+\left(N(r, w)-N_{\langle\mathrm{SS} 2\rangle}(r, w)\right)=S(r, w),
$$

then $w(z)$ satisfies a first order differential equation of the form (2.1).

The proof of Lemma 2 is given in [4].

LEMMA 3. Suppose the differential equation

$$
u^{\prime}\left(u^{\prime}+\eta(z) u^{2}\right)^{2}=b_{1}(z) u^{5}+\cdots+b_{5}(z) u+b_{6}(z)
$$

possesses an admissible solution $u(z)$. If $u(z)$ satisfies

$$
N_{1}(r, u)+m(r, u)=S(r, u),
$$

then $u(z)$ satisfies the Riccati equation or an equation of the form (2.1).

ProOF. We write (2.6),

$$
U\left(z, u, u^{\prime}\right)^{2}=V\left(z, u, u^{\prime}\right)
$$

where

$$
U\left(z, u, u^{\prime}\right)=u^{\prime}+\eta(z) u^{2}, \quad V\left(z, u, u^{\prime}\right)=\left[b_{1}(z) u^{5}+\cdots+b_{5}(z) u+b_{6}(z)\right] / u^{\prime} .
$$

Let $\mathscr{M}$ be the collection of coefficients of $U\left(z, u, u^{\prime}\right)$ and $V\left(z, u, u^{\prime}\right)$. Let $z_{0}$ be an admissible (w.r.t. $\mathscr{M}$ ) simple pole of $u$, and write in the neighbourhood of $z_{0}$,

$$
u(z)=\frac{R}{z-z_{0}}+\alpha+O\left(z-z_{0}\right) .
$$

Since the order of pole of $V(z)=V\left(z, u(z), u^{\prime}(z)\right)$ at $z_{0}$ is at most three, by (2.8), the order 
of pole of $U(z)=U\left(z, u(z), u^{\prime}(z)\right)$ at $z_{0}$ is at most one. Thus we have $-R+\eta\left(z_{0}\right) R^{2}=0$. Hence $R$ is written by small function, that is, $R=1 / \eta\left(z_{0}\right)$. For the sake of brevity, we put $R(z)=1 / \eta(z)$ in this proof.

First we treat the case $N(r, U)=S(r, u)$. From (2.7), we have

$$
\begin{aligned}
m(r, U) & \leq m\left(r, u^{\prime} / u\right)+m(r, u)+m\left(r, u^{2}\right)+S(r, u) \\
& \leq 3 m(r, u)+S(r, u) \leq S(r, u) .
\end{aligned}
$$

Hence $U(z)$ is a small function with respect to $u(z)$. Therefore $u(z)$ satisfies the Riccati equation in this case.

Secondly we treat the case $N(r, U) \neq S(r, u)$. We show that almost all admissible poles of $u(z)$ are simple poles of $U(z)$. By (2.7), we have to consider merely simple poles of $u(z)$.

We denote by $n^{*}(r, u)$ the number of admissible simple poles of $u(z)$ in $|z| \leqq r$ which are regular point of $U(z) . N^{*}(r, u)$ is defined in the usual way. Suppose $N^{*}(r, u) \neq S(r, u)$. There exists an admissible simple pole $z_{1}$ of $u(z)$, which is a regular point of $U(z)$. The order of pole of left-hand side of (2.6) at $z_{1}$ is at most two. If $\left|b_{1}\right|+\left|b_{2}\right|+\left|b_{3}\right| \neq 0$, then by the definition of admissible pole, the order of pole of right-hand side of (2.6) at $z_{1}$ is at least three, which is a contradiction. Thus $b_{1}(z)=b_{2}(z)=b_{3}(z) \equiv 0$ in $(2.6)$. Hence, by (2.6) $N(r, U)=S(r, u)$, which is a contradiction. Therefore, $N^{*}(r, u)=S(r, u)$ which implies that almost all admissible simple poles of $u(z)$ are simple poles of $U(z)$.

Let $z_{0}$ be an admissible simple pole of $u(z)$ and simple pole of $U(z)$. The order of pole of left-hand side of $(2.6)$ at $z_{0}$ is four. If $b_{1}(z) \not \equiv 0$, then by the definition of admissible pole, the order of pole of right-hand side of (2.6) at $z_{0}$ is five, which is a contradiction. Thus $b_{1}(z) \equiv 0$, and from the above estimation, we have $b_{2}(z) \not \equiv 0$. By simple calculation in the neighbourhood of $z_{0}$,

$$
\begin{aligned}
& V(z)=\frac{q\left(z_{0}\right)}{\left(z-z_{0}\right)^{2}}+\frac{p_{1}\left(z_{0}\right)+p_{2}\left(z_{0}\right) \alpha}{z-z_{0}}+O(1), \\
& \frac{q(z)}{R(z)} u^{\prime}(z)=-\frac{q\left(z_{0}\right)}{\left(z-z_{0}\right)^{2}}+\frac{p_{3}\left(z_{0}\right)}{z-z_{0}}+O(1),
\end{aligned}
$$

where $q(z)=-b_{2}(z) R(z)^{3}, \quad p_{1}(z)=-b_{2}^{\prime}(z) R(z)^{3}-b_{3}(z) R(z)^{2}, \quad p_{2}(z)=-4 b_{2}(z) R(z)^{2} \quad$ and $p_{3}(z)=-\left(q^{\prime}(z) R(z)-q(z) R^{\prime}(z)\right) / R(z)$.

Hence near $z_{0}$

$$
V(z)+\frac{q(z)}{R(z)} u^{\prime}-\frac{p_{1}(z)+p_{3}(z)}{R(z)} u=\frac{p_{2}\left(z_{0}\right) \alpha}{z-z_{0}}+O(1)
$$

We have

$$
U(z)+\frac{R^{\prime}(z)}{R(z)} u=\frac{2 \alpha}{z-z_{0}}+O(1)
$$


From (2.10) and (2.11), put

$$
\begin{aligned}
\varphi(z)= & 2\left[V\left(z, u, u^{\prime}\right)+\frac{q(z)}{R(z)} u^{\prime}-\frac{p_{1}(z)+p_{3}(z)}{R(z)} u\right] \\
& -p_{2}(z)\left[U\left(z, u, u^{\prime}\right)+\frac{R^{\prime}(z)}{R(z)} u\right]
\end{aligned}
$$

then $\varphi(z)$ is regular at $z_{0}$.

By (2.8), $V(z)$ is regular at zero of $u^{\prime}(z)$. Thus, we have $N(r, \varphi)=S(r, u)$. From (2.7) and (2.8),

$$
\begin{aligned}
m(r, \varphi) & \leq m(r, V)+m(r, U)+4 m(r, u)+S(r, u) \\
& \leq 3 m(r, U)+4 m(r, u)+S(r, u) \leq S(r, u) .
\end{aligned}
$$

Hence $\varphi(z)$ is a small function with respect to $u(z)$. From (2.8) and (2.12), $u(z)$ satisfies an equation of the form (2.1).

Q.E.D.

\section{Proof of Theorem 1.}

Put $u=1 /(w-\eta(z))$ in (1.4). Then by simple calculation (see [3])

$$
\begin{aligned}
& \beta_{1}(z) u^{\prime} u^{4}+\beta_{2}(z) u^{\prime 2} u^{2}+\beta_{3}(z) u^{\prime 3} \\
& =\Phi(z) u^{6}+b_{1}(z) u^{5}+\cdots+b_{5}(z) u+b_{6}(z),
\end{aligned}
$$

where

$$
\begin{gathered}
\beta_{k}(z)=(-1)^{k} \sum_{j=k}^{3}\left(\begin{array}{l}
j \\
k
\end{array}\right) \alpha_{j}(z) \eta^{\prime}(z)^{j-k}, \quad \alpha_{3}(z) \equiv 1, \quad k=0,1,2,3, \\
b_{i}(z)=\sum_{j=i}^{6}\left(\begin{array}{l}
j \\
i
\end{array}\right) a_{j}(z) \eta(z)^{j-i}, \quad i=0,1, \cdots, 6, \\
\Phi(z)=b_{0}(z)-\beta_{0}(z)=\sum_{j=0}^{6} a_{j}(z) \eta(z)^{j}-\sum_{j=0}^{3} \alpha_{j}(z) \eta^{\prime}(z)^{j}
\end{gathered}
$$

We assume that $\eta(z)$ is a small solution of (1.4). Thus we have $\Phi(z) \equiv 0$ in (3.1). For the proof of Theorem 1, we show that $w(z)$ satisfies (2.1) under the condition that $\eta(z)$ is neither deficient nor ramified small function w.r.t. $w(z)$, that is

$$
m(r, u)+N_{1}(r, u)=S(r, u) .
$$

Let $z_{0}$ be an admissible simple pole of $u(z)$. Write $u(z)$ near $z_{0}$ as:

$$
u(z)=\frac{R}{z-z_{0}}+\alpha+\beta\left(z-z_{0}\right)+\gamma\left(z-z_{0}\right)^{2}+\delta\left(z-z_{0}\right)^{3}+O\left(z-z_{0}\right)^{4} .
$$


From (3.1) and (3.3), since $\Phi(z) \equiv 0$,

$$
\begin{gathered}
\beta_{1}\left(z_{0}\right) R^{2}-\beta_{2}\left(z_{0}\right) R+\beta_{3}\left(z_{0}\right)=0, \\
{\left[4 \beta_{1}\left(z_{0}\right) R-2 \beta_{2}\left(z_{0}\right)\right] \alpha=P_{1}\left(R ; z_{0}\right),} \\
{\left[3 \beta_{1}\left(z_{0}\right) R^{2}-3 \beta_{3}\left(z_{0}\right)\right] \beta=P_{2}\left(R, \alpha ; z_{0}\right),} \\
{\left[2 \beta_{1}\left(z_{0}\right) R^{2}+2 \beta_{2}\left(z_{0}\right) R-6 \beta_{3}\left(z_{0}\right)\right] \gamma=P_{3}\left(R, \alpha, \beta ; z_{0}\right),} \\
{\left[-\beta_{1}\left(z_{0}\right) R^{4}-4 \beta_{2}\left(z_{0}\right) R^{3}+9 \beta_{3}\left(z_{0}\right) R^{2}\right] \delta=P_{4}\left(R, \alpha, \beta, \gamma ; z_{0}\right),}
\end{gathered}
$$

where $P_{j}\left(\cdot ; z_{0}\right)(j=1,2,3,4)$ are polynomials of corresponding arguments with small coefficients.

Since $\left|a_{6}\right|+\left|a_{5}\right|+\left|a_{4}\right|+\left|a_{3}\right| \not \equiv 0$, the right-hand side of (3.1) does not vanish. Thus we have

$$
\left|\beta_{1}\right|+\left|\beta_{2}\right|+\left|\beta_{3}\right| \not \equiv 0 \text {. }
$$

First we treat the case $\beta_{1}(z) \equiv 0$ or $\beta_{3}(z) \equiv 0$.

If $\beta_{1}(z) \equiv 0$, then we have $\beta_{2}(z) \not \equiv 0$ and $\beta_{3}(z) \not \equiv 0$. For, if $\beta_{2}(z) \equiv 0\left(\beta_{3}(z) \equiv 0\right)$, then by (3.4) $\beta_{3}\left(z_{0}\right)=0\left(\beta_{2}\left(z_{0}\right)=0\right)$. By Remark 1 , we have $\beta_{3}(z) \equiv 0\left(\beta_{2}(z) \equiv 0\right)$, which contradicts (3.9). Hence by (3.4) and (3.5), $R$ and $\alpha$ are written by small functions, which implies that $u(z)$ satisfies the Riccati equation (see [9], pp. 47-48).

Similarly to the case $\beta_{1}(z) \equiv 0$, if $\beta_{3}(z) \equiv 0$, then $\beta_{1}(z) \not \equiv 0$ and $\beta_{2}(z) \not \equiv 0$, and we obtain that $u(z)$ satisfies the Riccati equation.

Secondly we treat the case $\beta_{1}(z) \not \equiv 0$ and $\beta_{3}(z) \not \equiv 0$.

If $\left(-\beta_{2}(z) / \beta_{1}(z)\right)^{2}-4\left(\beta_{3}(z) / \beta_{1}(z)\right) \equiv 0$, that is, $\beta_{2}(z)^{2}-4 \beta_{1}(z) \beta_{3}(z) \equiv 0$, then the form of (3.1) is of the form (2.6). Thus by Lemma $3, u(z)$ satisfies the Riccati equation or an equation of the form (2.1).

Hence, in the below, we assume that $\beta_{2}(z)^{2}-4 \beta_{1}(z) \beta_{3}(z) \not \equiv 0$.

If any one of $\alpha, \beta, \gamma$ and $\delta$ is not written by the linear transformations of $R$ with small (w.r.t. $u(z)$ ) coefficients, that is, if $4 \beta_{1}\left(z_{0}\right) R-2 \beta_{2}\left(z_{0}\right)=0,3 \beta_{1}\left(z_{0}\right) R^{2}-3 \beta_{3}\left(z_{0}\right)=0$, $2 \beta_{1}\left(z_{0}\right) R^{2}+2 \beta_{2}\left(z_{0}\right) R-6 \beta_{3}\left(z_{0}\right)=0$ or $-\beta_{1}\left(z_{0}\right) R^{4}-4 \beta_{2}\left(z_{0}\right) R^{3}+9 \beta_{3}\left(z_{0}\right) R^{2}$ in (3.5)(3.8), then by (3.4), $\beta_{2}\left(z_{0}\right)^{2}-4 \beta_{1}\left(z_{0}\right) \beta_{3}\left(z_{0}\right)=0$ for each case. Hence by Remark 1 , $\beta_{2}(z)^{2}-4 \beta_{1}(z) \beta_{3}(z) \equiv 0$, which contradicts our assumption.

Here we have that for any admissible simple poles $z_{0}, \alpha, \beta, \gamma$ and $\delta$ are written by linear transformations of $R$ with coefficients of small (w.r.t. $u(z))$ functions. Thus, almost all admissible poles are SS2-kind poles. Hence by (3.2) and Lemma $2, u(z)$ satisfies the Riccati equation or a differential equation of the form (2.1). Thus by Remark 2, $w(z)$ satisfies a differential equation of the form (2.1). Therefore by Lemma 1, (1.4) is reducible, which implies that Theorem 1 is proved.

We would like to thank the refree for many comments and suggestions. 


\section{References}

[ 1 ] A. E. Eremenko, Meromorphic solutions of algebraic differential equations, Russian Math. Surveys, 37-4 (1982), 61-95.

[2] W. K. Hayman, Meromorphic Functions, Oxford Univ. Press, 1964.

[3] K. IshizaKI, Deficient and ramified small functions for admissible meromorphic solutions of some differential equations, Complex Variables Theory Appl., 13 (1989), 173-183.

[4] K. Ishizaki, Admissible solutions of some second order differential equations, Tech. Rep. Math. Sci. Chiba Univ., 6 (1990).

[5] K. Ishizaki and K. Funta, Admissible solutions of some algebraic differential equations $P\left(z, w^{\prime}\right)=Q(z, w)$, Res. Rep. of T.N.C.T., 21 (1989), 19-28.

[6] G. JANK and L. VolkmanN, Meromorphe Functionen und Differentialgleichungen, Birkhäuser, 1985.

[ 7 ] A. Z. Mokнońko and V. D. Mokнońko, Estimates for the Nevanlinna characteristic of some classes of meromorphic functions and their applications to differential equations, Sib. Math., J., 15 (1974), 921-934.

[ 8] R. Nevanlinna, Analytic Functions, Springer, 1970.

[9] N. Steinmetz, Ein Malmquistscher Satz für algebraische Differentialgleichungen erster Ordnung, J. Reine Angew. Math., 316 (1981), 44-53.

Present Address:

Department of Mathematics, Tokyo National College of Technology

KunUgida-CHO, HACHIOJI-SHI, TOKYO 193, JAPAN 